\author{
E. C. Carterette, University of California at Los Angeles, Presider
}

\title{
A minicomputer program for control and data acquisition in classical conditioning*
}

\author{
J. R. MILLENSON \\ Oxford Universitn: Oxford, England \\ and

\section{E. JAMES KEHOE, ROBERT W. TAIT and I. GORMEZANO \\ University of Iow'a, lowa City, lowa 52240}

\begin{abstract}
A 4K PDP-8/E computer program package has been developed to control classical conditioning procedures, to collect response data, and to extract statistical summeries. In contrast with other existing behavioral control languages geared to digital data typical of discrete operant analyses, this program distinguishes itself by its ability to retrieve and analyze the analog data arising from phasic response systems such as the rabbit's nictitating membrane. By means of a question-and-answer Teletype conversation with the $\mathrm{E}$, the program establishes trial sequience and trial spacing parameters; CS and US duration, probability, and interstimulus interval; intertrial interval fractionation for recording intertrial response frequencies; and session length. Various versions of the program exist to compute statistical properties of the analog response data, to dump detailed trial-by-trial topographies, and to attach instrumental contingencies to subtle features of the real-time analog responding.
\end{abstract}

The collection and quantification of analog response data in the computer control of Pavlovian conditioning preparations pose a number of problems outside the sope of current experimental control software. The commercial languages-Lehigh Valley's ACT, Digital Equipment Corporation's (DEC) SKED, and Grason-Stadler's SCAT-are oriented toward control of operant contingencies involving discrete response systems; hence, their exclusive concern with data which arrives at the computer in digital form. DEC's DATAK, which incorporates analog-to-digital (A/D) recording capabilities. possesses no stimuli control functions and requires the addition of DECtape for high-speed data collection.

*The program described herein was written in the summer of 1972. while the first author was on a leave of absence from Oxford University to the University of Iowa. Iowa City. The creation of the program and the preparation of this report were supported in part by NSF Grant GB-7907X to I. Gormezano and by SRC Grant B/SR5528 to J. R. Millenson.
In classical conditioning experiments, not only must a pattern of stimulus events be repeatedly generated. but resolution of a single response topography requires frequent measurement of a continuous response variable (e.g., skin resistance, blood pressure. eyelid closure) over a brief period of time. Consider the rabbit's nictitating membrane response (NMR) coupled to the transducing-recording system developed by Gormezano (1966). Briefly. membrane movement is monitored by connecting a nylon loop, sutured in the rabbit's membrane, to a hook joined by means of a thread to a counterbalanced rod. The rod is at tached to the axle of a rotary potentiometer and. following amplification of the electrical signal from the transducer, an analog record is obtained on a six-channel ink-writing oscillograph.

An oscillngraph record for a single conditioning trial. constituting the "raw" data, is pictured in Fig. 1. The upward deflection of the top line represents the duration of a $525-\mathrm{msec} 1.000-\mathrm{Hz}$ tone $\mathrm{CS}$; the downward deflection of the bottom line represents the duration of a 50-msec 4-mA paraorbital shock US; and the middle curve represents the analog record of membrane movement. Although Fig. 1 depicts the data of only a single $S$, we routinely obtain such analog records for six Ss simultaneously on every experimental trial.

For analysis, the following information is abstracted from such an oscillograph record. First, a conditioned response (CR) is identified by an upward deflection in

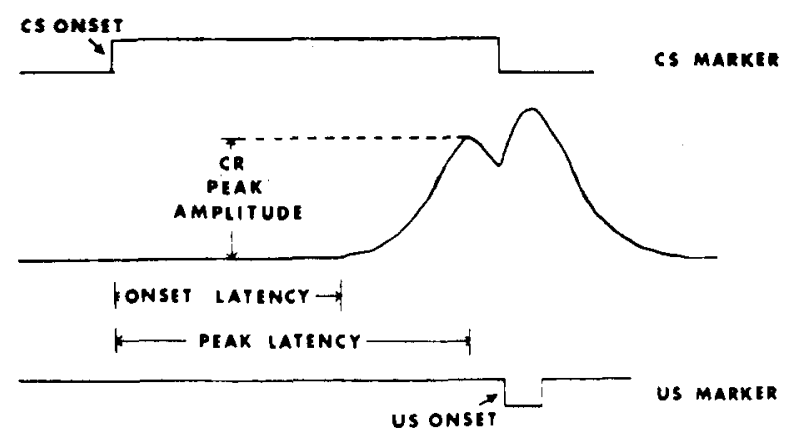

Fig. 1. Oscillograph record of CS and US occurrences and the topography of a nictitating membrane $C R$. 


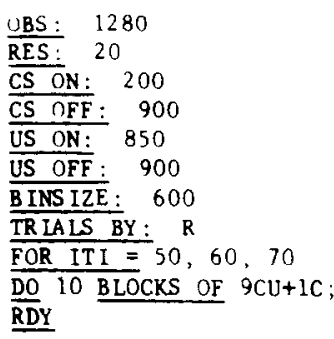

Fig. 2. Example of a session preamble conversation from the assembler. In this example, the RULE option is employed. The boldface (underlined) portions are produced by the assembler, the remaining portions are replies by the user.

the response line of more than $1 \mathrm{~mm}$ from baseline in the interval between CS onset and US onset. Given an amplification factor of two, a $1-\mathrm{mm}$ pen deflection thus represents a $1 / 2-\mathrm{mm}$ movement of the membrane. Second, the CR onset latency, peak amplitude, and peak latency are determined. The onset latency is specified by measuring the distance between onset of the CS and the point at which the upward deflection appears to begin. Peak amplitude and peak latency are determined by additional ruler measurements. Hitherto, these data have been laboriously transcribed by hand to data sheets, and subsequently keypunched on cards in preparation for summary analysis and statistical treatment by the university computer center batch processor.

The volume of data involved and the need for more flexible and sophisticated manipulations of experimental parameters attracted us to minicomputer control. In the design of a general program to accommodate our needs, we were guided and constrained by three considerations. First, the program was designed to collect a large amount of analog data and to abstract from these data the primary dependent measures routinely used in the analysis of classical NMR conditioning in the Iowa laboratory. Second. it was desirable that the program be able to simulate any of our usual stimulus presentation procedures previously generated by conventional modular control apparatus. The third consideration was a constraint on program capabilities due to the relatively small amount of core memory available in the current configuration. The $4 \mathrm{~K}$ of memory available had to contain the program and yet intake sufficient data to reflect faithfully the analog records exemplified by Fig. 1. Moreover, since no bulk backup storage was available, core memory had to be utilized to hold data records for sufficient time to be analyzed and recorded on punched paper tape.

\section{HARDWARE CONFIGURATION}

The lowa computer configuration consists of a PDP.8/E with a $4 \mathrm{~K}$ memory, an ASR33 Teletype, 16 channels of $A / D$ (an AD 8/E and AM8-EA) multiplexed by an AM8-EA controller, a DK8-EC real-time clock. two DR8-EA 12-bit digital input/output (I/O) buffers. and a high-speed paper-tape reader/punch. In addition. there is a $60-\mathrm{Hz}$ line-frequency clock and a 3-bit hardware random number generator (cf. Millenson \& Sullivan. 1969).

In the present system, the digital outputs to any stimulus device are wired through relay drivers to a patch panel. In this way, usage of the digital outputs is maximally flexible. As presently written, the program assumes one channel of analog data from each of six $\mathrm{S}$ stations. The software package is, however, being expanded to utilize 12 of the 16 available A/D channels, enabling concomitant measurement of analog signals from two independent response systems for each $\mathbf{S}$.

\section{GENERAL DESIGN}

Because of memory limitations, the various features of the classical conditioning software package had to be partitioned into three versions, designated $\mathrm{A}, \mathrm{B}$, and $\mathrm{C}$. The three versions differ primarily in the type of data which they collect. In addition, Version C permits modification of the US and presentation of other stimulus events contingent upon various parameters of the recorded $C R$. All three versions of the program consist of two major routines. An assembler processes the parameter specifications entered from the Teletype keyboard prior to the session, and a real-time clock-driven interrupt operating system carries out the actual control of the experimental session.

\section{The Assembler}

The assembler's properties can be best illustrated by examining preambles to typical conditioning sessions which are produced by a Teletype conversation between the assembler and the operator. The assembler obtains experimental parameters by producing a series of queries on the Teletype, to which the operator replies. In assembler mode, no experimental control takes place. because the operating system is disengaged by disabling all interrupts.

Figure 2 presents an example of a session preamble conversation from the assembler with the underlined portions indicating the characters produced by the assembler and remaining portions illustrating replies by the user. The program starts with a query. "OBS:," for observation interval. The operator replies with the length of an ubservation interval from 1 to $4.095 \mathrm{msec}$ and delimits this number with a carriage return. The observation interval defines a "trial" by specifying the length of time during which $A / D$ response measurements are to be taken. In conventional use. A/D measurements are initiated a short time before CS onset and continue for a time after US offset.

The query "RES: " for resolution interval. follows. This is a demand for the interval in milliseconds between successive readings of the input channels from the $S$ stations. The size of this interval is limited by the size of the buffer which stores the readings. At present. I maximum of 64 readings per station may be taken during an! observation interval. Thus, as the length of 
the observation interval increases, the minimum resolution unit must also increase. The assembler will test the resolution unit against the observation interval. If the resolution unit is too small, i.e., requiring more than 64 readings in the observation interval, the assembler will reissue the query "RES:" permitting the user to specify a new, larger resolution unit.

The next query, "CS ON:," asks for the time at which the $\mathrm{CS}$ is to come on relative to the beginning of the observation interval. In the example of Fig. 2, the CS is to begin $200 \mathrm{msec}$ after the beginning of the observation interval. One proceeds in a similar fashion to specify the offset of the CS, the onset of the US, and the offset of the US. Onset times and offset times cannot exceed the length of the observation interval, nor can an offset time be shorter than the onset time. Should any of the above constraints be violated, the assembler will reissue the queries for onset and offset times.

The query "BINSIZE:" follows the CS and US specification. BINSIZE requests specification of the size of the time interval into which the period between observation intervals, i.e., the intertrial interval (ITI), is to be divided. BINRESP, the routine which detects and records ITI responding, is controlled by the line-frequency clock. This routine requires that the size of the time bins be specified in $16.67-\mathrm{msec}$ units. In the example of Fig. 2, the bin size is specified at 600; thus, the ITI will be broken into $10-\mathrm{sec}$ units. An ITI can be broken into a maximum of eight bins. If the bin size is too small, requiring more than eight bins, no error is indicated. Instead, the eighth bin will be automatically lengthened to occupy the remaining portion of the ITI. If the ITI is variable, as it usually is, BINSIZE is ordinarily specified such as to divide the longest possible ITI into eight equal-sized bins. The shorter ITIs will not use all the bins, but BINRESP will indicate at printout time how many times each bin is used in the course of a session.

Following the delineation of trial parameters, the trial sequence is specified by selecting one of three routines by replying with the routine's initial letter to the query "TRIALS BY:." The routines are RULE, PROBABILITY, and TAPE.

All three routines recognize the following characters: (1) $\mathrm{C}$ for $\mathrm{CS}_{1}$, (2) $\mathrm{X}$ for $\mathrm{CS}_{2}$, (3) U for US, and (4); (semicolon) for topographical data collection. There are additional characters idiosyncratic to each routine. Should an illegal character be encountered, the routine will recommence.

In practice, RULE has been the most widely used option. It is employed in a conditioning situation in which a sequence of CS-US pairings is followed by a CS-alone test trial. For example, a 100-trial session with ITI values at 50,60 , and $70 \mathrm{sec}$ and consisting of 10 blocks of 9 paired trials followed by 1 test trial is specified, as indicated in the figure, in the following fashion:
FOR ITI $=50,60,70$

DO 10 BLOCKS OF $9 \mathrm{CU}+1 \mathrm{C}$;

(The boldface portions of the above statement are produced by the assembler; the remainder is filled in by the user.) The ITI values are specified in seconds. Up to eight ITI values may be delineated. The assembler will then randomize the specified values across trials, subject to the restriction that no more than three ITIs of the same value may occur in succession. The semicolon indicates that special analyses are to be performed on the test trial data.

Upon completion of assembly, a ready message, "RDY," is generated by the assembler. The operator can then start the session by striking the carriage return key on the Teletype, which brings the program into the operating system mode by enabling interrupts.

The second trial sequencing option, PROBABILITY, produces an exponential distribution of ITI values. In all other respects, PROBABILITY is identical to RULE. To produce the ITI distribution, the operator specifies a minimum ITI value and defines the constant probability with which a trial will occur after the minimum interval has elapsed. The trial sequence parameters are specified in the following fashion:

\section{FOR MIN ITI $=40$, WITH P $=1 / 8\left(\mathrm{AV} .=320^{\prime \prime}\right)$ DO 10 BLOCKS OF $9 \mathrm{CU}+1 \mathrm{C}$;}

The probability's divisor must be a power of 2 . The minimum probability is $1 / 64$.

RULE and PROBABILITY recognize an additional character, "V," for variable CS duration. Should this character be encountered, the assembler then asks "CS'S," to which the user may reply with up to eight values in milliseconds. The proportion of CSs of different durations may be varied by repeating values. Take a schedule, for example, in which one desires CS durations of 200 and $700 \mathrm{msec}$ in a 3 to 1 proportion. This schedule would be specified in the following manner: CS'S: 200, 200, 200, 700. Specification of variable-duration CSs will automatically produce a delayed conditioning paradigm in which the CS terminates with US onset, thereby producing variable-length interstimulus intervals (ISIs). The CS OFF and US ON parameters specified earlier in the preamble are ignored, although the US duration is computed from the difference between US ON and US OFF.

The third option, TAPE, conforms closely to the conventional manner of trial sequencing from a coded tape, enumerating the exact ITI value for each and every trial. For this routine, the user types in sequence an ITI value specified in seconds, followed by the characters denoting the events which are to occur on the following trial. The additional characters recognized by TAPE are the comma, the backward arrow, and the dollar sign. For 
Table 1

Allocation of Core in "A" Version

\begin{tabular}{|c|c|}
\hline Page & Rout ines \\
\hline$\infty 00$ & CONSTANTS AND POINTER ADDRESSES \\
\hline 200 & ASSEMBLER \\
\hline $\begin{array}{l}400 \\
600\end{array}$ & RULE OPTION OF ASSEMBLER \\
\hline $\begin{array}{l}1000 \\
1200\end{array}$ & PROBAB ILITY OPTION OF ASSEMBLER \\
\hline $\begin{array}{l}1400 \\
1600 \\
2000\end{array}$ & $\begin{array}{l}\text { OPERATING SYSTEM AND } \\
\text { INTERRUPT SERVICE ROUT INES }\end{array}$ \\
\hline $\begin{array}{l}2200 \\
2400 \\
2600 \\
3000 \\
3200\end{array}$ & $\begin{array}{l}\text { STORAGE BUFFER FOR } \\
\text { AVERAGED RES PONSE TOPOGRAPHIES } \\
\text { CALCULATED BY GETSTATS }\end{array}$ \\
\hline $\begin{array}{l}3400 \\
3600 \\
4000\end{array}$ & $\begin{array}{l}\text { TEMPADAREA, } \\
\text { STORAGE BUFFER FOR DATA } \\
\text { COLLECTED BY ADIN }\end{array}$ \\
\hline $\begin{array}{l}4200 \\
4400\end{array}$ & ADIN \\
\hline 4600 & TAPE OPTION OF ASSEMBLER \\
\hline $\begin{array}{l}5000 \\
5200 \\
5400 \\
5600\end{array}$ & GETSTATS \\
\hline $\begin{array}{l}6000 \\
6200 \\
6400 \\
6600\end{array}$ & $\begin{array}{l}\text { LIBRARY OF SUBROUT INES FOR } \\
\text { ARTTKMETIC, PRINTING, PUNCHING, AND READING }\end{array}$ \\
\hline $\begin{array}{l}7000 \\
7200 \\
7400 \\
7600\end{array}$ & $\begin{array}{l}\text { LADERS AND SUMMARY STATTSTICS } \\
\text { CALCULATED BY GETSTATS }\end{array}$ \\
\hline
\end{tabular}

\footnotetext{
NOTF:--Routines unique to " $B$ " and " $C$ " verslons occupy the pages utilized by GETSATS and its storage buffer in the " $A$ " version.
}

example, a differential conditioning session consisting of 30 trials could be specified in the following fashion:

$$
\begin{aligned}
& 50 C U, 60 X, 50 C U, 70 C U, 40 X, 70 X, 40 C U, 70 X \\
& 60 C U, 60 X \leftarrow \leftarrow \$
\end{aligned}
$$

The two-digit number preceding each letter combination is the ITI value in seconds prior to the trial. In this case, $\mathrm{C}$ stands for the CSt and X for the CS-. Both C and X are currently programmed to follow the same temporal parameters specified earlier in the preamble. There is no restriction on the combination of events in any trial. For instance, "CXU" would be a legal combination, producing a compound stimulus.

The comma, the semicolon, and the dollar sign are the trial delimiters. The comma delimits an ITI-trial pair and requests that the onset latency for any CRs be collected for the delimited trial. The semicolon indicates that special data analyses are to be performed upon any CRs occurring on that trial.

The number of backward arrows prior to the dollar sign indicate the number of times the sequence is to be repeated after the first execution of the sequence. One may have as many as 63 repeat arrows and as many as 63 trials per sequence. The dollar sign is the end-of-sequence delimiter.

\section{The Operating System}

The basis of the operating system (OPSYST) is a three-device interrupt skip chain. The three devices causing interrupts are the Teletype keyboard, the line-frequency clock, and the $500-\mathrm{Hz}$ clock. When not servicing interrupts, OPSYST executes routines which perform background data analysis or data output.

The Teletype interrupt service routine permits the $\mathrm{E}$ to suspend the experimental session and restart it at one of several points. Typing "CTRL" and " $Z$ " characters will suspend the program and display on the PDP-8/E console the current reading from the analog input channel selected by the setting of the switch register. This suspend facility permits adjustment of $A / D$ balancing voltages at any time during the session to compensate for drifting response baselines. Setting the leftmost bit of the switch register resumes the experiment at the point where it was suspended. The "CTRL" and "F" characters will clear the data storage registers and start the session from the beginning with the same parameters as specified in the assembler, a useful facility when several squads of Ss are run under identical experimental conditions. The "CTRL" and " $K$ " characters will restart the program from the beginning of the assembler with previous parameters erased.

Conceptually, OPSYST performs two functions: stimulus generation and data collection. Stimulus generation is a straightforward matter of turning on and off stimuli at appropriate times determined by the $500-\mathrm{Hz}$ crystal clock interrupt service routine. The data collection function is subdivided into real-time collection routines and background analytic routines. Two routines, ADIN and BINRESP, perform data collection functions. ADIN operates during the observation interval and is triggered by the $500-\mathrm{Hz}$ clock at the appropriate resolution units; BINRESP operates between observation intervals and is governed by the line-frequency clock. GETSTATS is the primary analytic routine and operates in background upon data already collected.

ADIN collects and stores analog data for further analysis. This routine also concerns itself with response detection in order to implement response-stimulus contingencies. ADIN samples each S station once every resolution unit. On each sample, ADIN stores the reading from each station in two locations. The first location, known as TEMPADAREA, retains all the readings from all stations for a single observation interval (see Table 1). As noted above, there may be as many as 
64 readings per station for an observation interval. In this way, a representation of the full response topography for a trial is stored for posttrial analysis. The second storage location consists of six registers containing the current sample. one reading from each station. These readings are used internally by ADIN for response detection.

ADIN detects responses by acting as a software comparator circuit. In the time between the onset of the observation interval and the onset of the first stimulus event (usually the CS) ADIN establishes the average value for each station and uses this value as a baseline against which to compare samples taken later in the observation interval. Should the baseline change by more than $1 \mathrm{~mm}$ during the preevent interval, ADIN still calculates the average value but will set a software flag indicating that a response is in progress. ADIN finds such baseline changes by comparing the largest and smallest readings taken during the preevent interval. If the difference between these readings exceeds the equivalent of $1 \mathrm{~mm}$ of movement, the software flag for the appropriate station is set.

After the onset of the first stimulus event of a trial, ADIN compares its readings against a response criterion of $1 / 2 \mathrm{~mm}$ of positive movement. Should a reading exceed the criterion, a software flag. RISEFLAG, will be set for the appropriate station. ADIN also detects the passage of response maxima. This is accomplished by comparing the current reading against the largest previously encountered reading for the ... in progress. Should the current reading fall $1 \mathrm{~mm}$ below the largest reading, another software flag, FALLFLAG, will be set for the appropriate station.

Operating between observation intervals and used by program version $B, B I N R E S P$. the second data collection program functions as a multilevel comparator circuit, storing the frequency of responses which it detects into appropriate intertrial interval bins. This routine examines each $\mathrm{A} / \mathrm{D}$ channel every $16.67 \mathrm{msec}$. If the second of two consecutive readings is greater than the first by a preset response amplitude criterion, a response is counted in one of the eight bins into which the ITI has been fractionated by the preamble parameter BINSIZE. Currently, the preset criterion corresponds to $1 / 8 \mathrm{~mm}$ of NMR movement, and ITI responses of multiples of 1,2 , and 4 (representing movements of $1 / 8,1 / 4$, and $1 / 2 \mathrm{~mm}$ ) of the criterion amplitude can all be independently collected.

A sample of BINRESP's output for an experimental session consisting of 100 trials presented at ITIs of 50 , 60 , and $70 \mathrm{sec}$ can be seen in Fig. 3. At the top of the output are listed the longest ITI, the size of the bins in seconds, and the frequency with which each bin was used in the course of the session. Only the output for a single station is shown in Fig. 3. The values of 006, 013, and 026 in the left column are the octal values which correspond to comparator levels of $1 / 8,1 / 4$, and $1 / 2 \mathrm{~mm}$ for the Iowa system. Listed next to each level is
II I $=0070$ B INS : 0010"

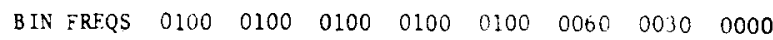

$\mathrm{B} \cdot \times 001$

$\begin{array}{lllllllll}006 & 0083 & 0015 & 0008 & 0008 & 0007 & 0009 & 0003 & 0000\end{array}$

$\begin{array}{lllllllll}013 & 0067 & 0006 & 0005 & 0003 & 0004 & 0007 & 0002 & 0000\end{array}$

$\begin{array}{lllllllll}026 & 0034 & 0003 & 0004 & 0002 & 0002 & 0004 & 0002 & 0000\end{array}$

Fig. 3. Example of the output of the BINRESP routine for recording intertiral responding. Only the data for a single $S$ is shown.

the frequency with which responses of the appropriate level were detected for each time bin in the course of the session.

The analytic routine GETSTATS, used by Version A of the program, computes the descriptive dependent variables described in Fig. 1. During the intertrial interval, this routine analyzes the data input by ADIN from the previous trial and signals the operator if any special conditions (see below) in the data are encountered. On ordinary trials, GETSTATS stores for each channel the number of responses, the sum of the onset latencies, and the sum of the squared onset latencies. On special trials designated by a semicolon in the trial event specification, GETSTATS performs additional analyses. It collects peak amplitudes and their squares, peak latencies and their squares, and response durations and their squares, thus providing all the information needed for standard statistical analyses. Furthermore, the entire set of readings for each channel is averaged with previous sets of readings. In this way, an average topography for each S's responding is obtained.

The summary statistics, such as onset latencies, but not the averaged topographies, are stored in one of three buffers according to which stimulus events were programmed on the trial. One buffer holds statistics derived from any trial on which a $\mathrm{C}$ is designated. These are the $\mathrm{C}, \mathrm{CU}, \mathrm{CX}$, and $\mathrm{CXU}$ combinations. A second buffer holds statistics derived from any trial on which an $\mathrm{X}$ but not a $\mathrm{C}$ is designated. These are the $\mathrm{X}$, and the $\mathrm{XU}$ combinations. The third buffer stores statistics derived from trials in which only a $U$ is designated. Thus, one can obtain UR as well as CR statistics.

GETSTATS signals the operator if it encounters any of three special data conditions! First, the pretrial baseline may be resting at an extreme value of the possible range. If the baseline value is too high, response features may be attenuated. Extreme readings indicate that the transducer balancing circuit requires adjustment. Second, a response may be occurring prior to the stimulus events. Such responding may obscure the $S$ 's reaction to the stimuli. Third, a failure to respond on a trial on which a US is scheduled may indicate an apparatus failure at the site of the experimental chamber. Upon detecting any of these problems, GETSTATS will ignore the data on the given trial and signal the operator by illuminating appropriate lights 
patched to the digital output buffer.

In the B version of the program, no statistical analysis is possible, for GETSTATS is incompatible with BINRESP. The $\mathrm{B}$ version does, however, contain a routine which dumps onto punched paper tape the "raw" response topographical data for trials designated with a semicolon. Like GETSTATS, the dumping routine operates as background in the OPSYST. Thus, one subsequent trial can continue to be presented while the data from the last trial is being dumped. With the 50 -character/sec punch, it is possible to dump out the topographic data from six Ss from a single trial in less than $30 \mathrm{sec}$.

The $C$ version of the program is intended to produce instrumental contingencies that may be applied to various features of the response topography measured by ADIN during the observational interval. To date, Version $C$ has been used only in conjunction with the RISEFLAG signaling that a response has been initiated to the CS. One routine of the $\mathrm{C}$ version delivers a single $300-\mathrm{msec}$ output pulse the first time a RISEFLAG is encountered on a trial. By means of a solenoid-driven water delivery system. this pulse is translated into the delivery of $1 \mathrm{cc}$ of water to each of three Ss and their yoked controls in studies of instrumental shaping of NMRs. Although not as yet explored, it is evident that a variety of more complex features of the response topography, including its rise and decay times, its time integral, and so forth, could be used to trigger external reinforcing events to test the susceptibility of such systems to contingent reward and punishment operations.

\section{CONCLUSIONS}

The classical conditioning software package described above is a program still under development. With regard to its stimulus control functions, separate specification of the temporal parameters of the $\mathrm{C}$ and $\mathrm{X}$ stimuli would be desirable. As for data collection, the program is currently oriented toward monophasic response systems which exhibit relatively steady baselines. Biphasic response systems, such as heart rate, which have fluctuating baselines would require individual and idiosyncratic GETSTATS routines.

Notwithstanding its limitations, the package has proved serviceable in a variety of conditions. It has been used to control experiments dealing with variable interstimulus intervals, with temporal conditioning, and with water reinforcement contingent upon a nictitating membrane CR. In carrying out these experiments, the program has collected and analyzed in real time topographic response data which previously would have required hundreds of manhours to obtain.

\section{REFERENCES}

Gormezano, I. Classical conditioning. In J. B. Sidowski (Ed.), Experimental methods and instrumentation in psychology. New York: McGraw-Hill, 1966.

Millenson, J. R., \& Sullivan, G. D. A hardware random number generator for use with computer control of probabilistic contingencies. Behavior Research Methods \& Instrumentation. 1969, 1. 194-196. 